\title{
Variational Methods in AdS/CFT
}

\author{
Tomás Andrade, Máximo Bañados and Francisco Rojas \\ Departamento de Física, \\ P. Universidad Católica de Chile, Casilla 306, Santiago 22, Chile. \\ tiandrad@uc.cl, maxbanados@fis.puc.cl, frojasf@uc.cl
}

\begin{abstract}
We prove that the $A d S / C F T$ calculation of 1-point functions can be drastically simplified by using variational arguments. We give a simple proof, valid for any theory that can be derived from a Lagrangian, that the large radius divergencies in 1-point functions can always be renormalized away (at least in the semiclassical approximation). The renormalized 1-point functions then follow by a simple variational problem involving only finite quantities. Several examples, a massive scalar, gravity, and renormalization flows, are discussed. Our results are general and can thus be used for dualities beyond $A d S / C F T$.
\end{abstract}

\section{Introduction and motivation}

In the AdS/CFT correspondence [1, 2, 3], the key object to be calculated is the generating functional $W[J]$ of connected diagrams associated to an operator $\mathcal{O}$. According to the proposal put forward in [1, 2, 3], for each operator $\mathcal{O}$ there exists a dual bulk field $\Phi$ with action $I[\Phi]$ such that

$$
e^{-W[J]}=\int_{A d S} D \Phi e^{-I[\Phi]}
$$


The integral in the right hand side depends on the source $J$ through boundary conditions. For a generic asymptotic expansion 1 ,

$$
\Phi(x, \rho) \rightarrow \rho^{\sigma_{0}} \varphi_{0}(x)+\rho^{\sigma_{1}} \varphi_{1}(x)+\rho^{\sigma_{2}} \varphi_{2}(x)+\cdots, \quad \rho \rightarrow 0 .
$$

where $\sigma_{0}<\sigma_{1}<\sigma_{2} \ldots$ The leading coefficient $\varphi_{0}(x)$ is identified with the source (see [4, 5] for other cases),

$$
J(x)=\varphi_{0}(x),
$$

and the path integral in (11) is computed with $\varphi_{0}(x)$ fixed.

The calculation of the integral in (1) is as difficult, if not more, as the CFT calculation of $W[J]$. What makes AdS/CFT useful is the fact that the correspondence is strong/weak. This means that the semiclassical approximation of (11),

$$
\left.W[J] \simeq I\left[\Phi\left(\varphi_{0}\right)\right]\right|_{\text {on-shell }},
$$

((13) is understood) gives strongly coupled results for $W[J]$.

Now, the approximation (4) is valid provided the action $I[\Phi]$ is stationary under variations with $\varphi_{0}(x)$ fixed, that is, the variation of the action must satisfy,

$$
\delta I[\Phi]=\int_{M} \mathcal{E}(L) \delta \phi+\int_{\partial M} A \delta \varphi_{0}
$$

where $A$ is some coefficient, and $\mathcal{E}(L)$ are the Euler-Lagrange equations. If the action $I[\Phi]$ fails to satisfy (5), then the semiclassical approximation is not valid and, of course, (44) is not valid either 2 .

The importance of the variational problem in AdS/CFT calculations was stressed in [6], where ambiguities in the holographic description of Dirac spinors [7] were fixed. Even for the simple example of massive scalars, problems with the Ward identities [8] were solved by a proper analysis of the variational problem [4, 9, 10]. Since then, the analysis of boundary terms in AdS/CFT, specially for scalar fields, has received much attention [10, 11, 12, 13, 14. More recently, this problem has revived in the context of multi trace deformations, e.g., [15, 16, 17, 18, 19, 20, 21].

\footnotetext{
${ }^{1} \sigma_{i}$ are typically rational numbers, and $\sigma_{0}$ is associated to the conformal dimension of the dual operator. $\rho$ is the radial coordinate $\rho \rightarrow 0$ defining the boundary. There may be logarithmic terms at some order which are relevant for the structure of the solution and for the anomalies. At this point, we only need the existence of some series.

${ }^{2}$ It sometimes argued that the AdS/CFT prescription is incomplete because boundary terms can be added to the action without affecting the equations of motion. This point of view is incorrect. The action functional $I[\Phi]$ is uniquely defined, up to trivial scheme dependent terms, once the source $\varphi_{0}$ is identified.
} 
Besides being stationary, the action $I[\Phi]$, and in particular the coefficient $A$ in Eq. (15), must be finite, otherwise the equality (4) would not be very useful. Since the background has infinite volume (normally AdS space, but other backgrounds are also relevant for realistic dualities) divergencies do arise and they need to be renormalized. This is done by standard technics. One first introduces a regulator and then subtracts divergent terms. As first noticed in [8, 9], it is necessary to first formulate a proper Dirichlet problem at at fixed value of the regulator, and take the limit at the very end. A general procedure, termed Holographic Renormalization, was put forward in [10], and a Hamiltonian approach was developed in [22. In particular, in [22, general formulas for Asymptotically locally AdS spacetimes have been displayed. Dimensional regularization is also available with equivalent results [24, 25, 26]. A Hamilton-Jacobi method, exploiting the gravitational Hamiltonian constraint, is also available [27].

The goal of this paper is to show that variational arguments can provide simplifications in explicit calculations. Variational approaches have been considered previously in the literature (see [23] and references therein), including the issue of global charges. Our goal here is to put forward a novel and simple approach to deal with "renormalization problem". We prove that the infinities can always be renormalized away, for any theory, at least in the semiclassical approximation (4). In particular, we prove that all divergencies appearing in the variation of the action can be subtracted without interfering with the finite parts. This means that the calculation of 1-point functions $\langle\mathcal{O}\rangle_{J}$ (in the presence of sources) can be done via a simple finite variational problem and divergencies can be ignored completely 3 .

Furthermore, our procedure does not make use of the AdS structure explicitly. Thus, in principle, it could be useful in more realistic gauge theory/gravity correspondences (see [29] for a recent discussion) where explicit calculations have been hampered by technical difficulties associated with the renormalization problem. Particular applications of the method described here have appeared in 30, 31.

\footnotetext{
${ }^{3}$ Of course divergencies contain important information and in some cases it is preferably to keep them, e.g., [28]. But, as far as the semiclassical 1-point function is concerned, we shall see that they can be ignored completely.
} 


\section{The variational problem and 1-point functions}

In this section we prove the main result of the paper, namely, that 1-point functions (with non-zero sources) can be computed in AdS/CFT without calculating the divergent terms.

Consider the general action (see Sec. 4 for a more general action)

$$
I[\Phi]=\int_{\epsilon} d \rho \int_{M} d^{d} x L\left(\Phi, \Phi^{\prime}, \partial_{i} \Phi\right)+\int_{\rho=\epsilon} d^{d} x B .
$$

with associated the Euler-Lagrange equations

$$
\left(\frac{\partial L}{\partial \Phi^{\prime}}\right)^{\prime}+\partial_{i}\left(\frac{\partial L}{\partial_{i} \Phi}\right)=\frac{\partial L}{\partial \Phi}
$$

The action is defined on a manifold with asymptotic topology $\Re \times M$. $\Phi$ denotes collectively the set of fields under consideration. $\rho$ is a radial coordinate and the boundary is located at $\rho \rightarrow 0$. We follow closely the notations introduced in [10]. The parameter $\epsilon$ is a cutoff that regularizes the eventual IR divergencies at $\rho \rightarrow 0$. The primes denote derivatives with respect to $\rho$, which we have separated from the other derivatives only for illustrative purposes.

The holographic calculation of field theory correlators dual to an action $I$ has three different faces. The dictionary problem establishes the relationship between bulk fields and dual operators. This is where most of the AdS/CFT physics resides. The renormalization and Dirichlet problems, namely, to find $B$ to make the bulk action finite and well-defined for the given boundary conditions. Finally, the fluctuation problem involves imposing boundary conditions in the deep interior and find non-local relations among the boundary data. In this paper we shall only deal with the second issue, namely, the renormalization problem. We refer to the large literature for the other problems.

As usual, we formulate the holographic renormalization problem as follows: We seek a boundary term $B$ such that the on shell variation of the action (6) satisfies

$$
\begin{aligned}
\delta I[\Phi] & =\lim _{\epsilon \rightarrow 0} \int_{\rho=\epsilon} d^{d} x\left(\frac{\partial L}{\partial \Phi^{\prime}} \delta \Phi+\delta B\right) \\
& =\int d^{d} x A \delta \varphi_{0}
\end{aligned}
$$

where $A$ is a finite coefficient, and $\varphi_{0}$ is the source for the given problem. If (8) can be written in the form (91), then, according to [2, 3], the problem is solved and the vev of the dual operator is

$$
\langle\mathcal{O}\rangle=A
$$


It is clear from the transition from (8) to (9) that the boundary term $B$ is playing two roles. On the one hand it must ensure that the Dirichlet problem is well defined, namely, $\delta I \sim \int A \delta \varphi_{0}$. On the other, we demand $A$ in (9) to be finite, and hence $B$ must remove the divergencies coming from the bulk. We show here that these two problems can in fact be analyzed separately.

\subsection{The renormalization problem.}

Let us first deal with renormalization problem. We prove here that all divergencies in $\frac{\partial L}{\partial \Phi^{\prime}} \delta \Phi$ can be written as total variations, and hence be canceled by $B$. This result does not depend on the explicit form of the series (2). To fix the ideas, we expand both $\Phi$ and $\delta \Phi$ in the form (see footnote 1),

$$
\begin{aligned}
\Phi & =\rho^{\sigma}\left(\varphi_{0}+\rho \varphi_{1}+\rho^{2} \varphi_{2}+\cdots\right) \\
\delta \Phi & =\rho^{\sigma}\left(\delta \varphi_{0}+\rho \delta \varphi_{1}+\rho^{2} \delta \varphi_{2}+\cdots\right) .
\end{aligned}
$$

and insert them in $\frac{\partial L}{\partial \Phi^{\prime}} \delta \Phi$. We find an expansion of the form,

$$
\frac{\partial L}{\partial \Phi^{\prime}} \delta \Phi=\sum_{n=-K}^{\infty} \rho^{n} C_{n}\left(\varphi_{i}, \delta \varphi_{j}\right)
$$

Here, $\varphi_{i}$ are the coefficients appearing in the series (11). $K$ is some positive number and represent the fact that, generically, (12) contains divergent pieces. There may be log's and other types of functions of $\rho$. As it will be clear in a moment, this will not be relevant for the analysis that follows.

We are now going to prove the following general result. All coefficients $C_{n}(\varphi, \delta \varphi)$, except the zero mode $C_{0}$, can be written as a total variation. This means, there exists local functions $D_{n}\left(\varphi_{i}\right)$ such that,

$$
C_{n}\left(\varphi_{i}, \delta \varphi_{j}\right)=\delta D_{n}\left(\varphi_{i}\right), \quad \forall n \neq 0
$$

To this end, consider the following equality,

$$
\begin{aligned}
\left(\frac{\partial L}{\partial \Phi^{\prime}} \delta \Phi\right)^{\prime} & =\left(\frac{\partial L}{\partial \Phi^{\prime}}\right)^{\prime} \delta \Phi+\frac{\partial L}{\partial \Phi^{\prime}} \delta \Phi^{\prime} \\
& =\left(\frac{\partial L}{\partial \Phi}-\partial_{i}\left(\frac{\partial L}{\partial_{i} \Phi}\right)\right) \delta \Phi+\frac{\partial L}{\partial \Phi^{\prime}} \delta \Phi^{\prime} \\
& =\frac{\partial L}{\partial \Phi} \delta \Phi+\left(\frac{\partial L}{\partial_{i} \Phi}\right) \delta\left(\partial_{i} \Phi\right)+\frac{\partial L}{\partial \Phi^{\prime}} \delta \Phi^{\prime}-\partial_{i}\left(\frac{\partial L}{\partial_{i} \Phi} \delta \Phi\right) \\
& =\delta L
\end{aligned}
$$


In the second line we have used the equations (77), and in the last line we have used that $L=$ $L\left(\Phi, \Phi^{\prime}, \partial_{i} \Phi\right)$. We have also discarded a total derivative term which, under the integral sign, $\int d^{d} x$, will not contribute.

Taking the radial derivative of (12) at both sides and comparing with (14) we conclude that (13) is in fact true, and that the functions $D_{n}$ are the Taylor coefficients of the Lagrangian $L$. Since divergencies are contained in $\rho$-dependent contributions, $1 / \rho^{n}, \log \rho$, etc, we conclude that they can always be subtracted by choosing $B$ appropriately. See sections 3.1 and 3.4 for explicit examples.

Note also that neither the form of the series (11) nor (12) are really relevant. The only important point is to distinguish between the $\rho$-dependent parts of $\frac{\partial L}{\partial \Phi^{\prime}} \delta \Phi$, and its zero mode. According to the above result, all $\rho$ - dependent parts are total variations, while no information can be extracted on the zero mode. This is a completely general result 4 valid also for higher order theories (see Sec. (4).

Summarizing, when computing the on-shell variation of the action (6) one can simply ignore all divergent terms and keep only the zero mode (in the $\rho$ expansion). The above result is general enough to ensure the existence of $B$ to subtract all divergencies and this is all one needs at this point. The 1-point functions are finite and only depend on the zero modes of $\frac{\partial L}{\partial \Phi^{\prime}} \delta \Phi$ and $B$. We now turn into their analysis.

\subsection{The variational problem}

Having discarded all divergencies by a choice of $B$, the problem (9) is turned into a restricted and much simpler problem, namely, to find $B_{0}$ such that,

$$
\begin{aligned}
\delta I & =\int d^{d} x\left(\left.\frac{\partial L}{\partial \Phi^{\prime}} \delta \Phi\right|_{\text {zero mode }}+\delta B_{0}\right), \\
& =\int d^{d} x A \delta \varphi_{0},
\end{aligned}
$$

where $B_{0}$ is now the zero mode part of $B$. The finite coefficient $A$ equals to the dual vev $\langle\mathcal{O}\rangle=A$. This problem does not have a universal solution and, in fact, $B_{0}$ may not even exist at all unless certain integrability condition is fulfilled.

\footnotetext{
${ }^{4}$ The formula (14) may also be relevant for an off-shell formulation of the action, and the finiteness of conserved charges. It is known that when turning on non-standard modes of some fields, divergencies in the charges are canceled after properly incorporating the back reaction into the boundary conditions [32, 33]. Presumably, these cancelations can be understood as special cases of (14). We thank M. Henneaux for a discussion on this point.
} 
It is worth noting that $A$ may contain purely local pieces, that cannot be eliminated unless they are integrable, as in the case of gravity (see Sec. 3.3).

We would like to end this paragraph remarking that this procedure to deal with the finite part of the on-shell variation of the action has already appeared in the literature in various examples, e.g., [11, 12, 13, 14, 20, 30, 31]. General formulae for asymptotically locally AdS spacetimes are also known [23].

\section{Examples}

We display in this section some examples and applications of the variational approach to the holographic calculation of correlators. We consider here a toy model, a massive scalar field, and gravity.

\subsection{Toy model and comparison with Holographic Renormalization}

Let us illustrate the ideas presented so far with a simple example. Consider the action for a toy model in $0+1$ dimensions,

$$
I=\frac{1}{2} \int d \rho\left[\frac{2}{\rho} \Phi^{\prime 2}-\frac{2 \kappa}{\rho^{2}} \Phi^{2}-\frac{3}{2 \rho^{3}} \Phi^{2}\right]+B
$$

This system has the same features of a scalar field on $A d S_{4}$ with dual conformal dimension $\Delta=3, \kappa$ playing the role of the Laplacian. Setting $\Phi=\rho^{1 / 2} \varphi(\rho)$, the equation of motion become $\rho \varphi^{\prime \prime}+\kappa \varphi=0$, which has the asymptotic series,

$$
\varphi=\varphi_{0}+\rho\left(\varphi_{1}+\psi \log \rho\right)+\cdots
$$

with $\varphi_{0}$ and $\varphi_{1}$ arbitrary while $\psi=-\kappa \varphi_{0}$. The exact solution exists in terms of Bessel functions. The on-shell variation of the action becomes,

$$
\begin{aligned}
\delta I & =\left.\frac{2}{\rho} \Phi^{\prime} \delta \Phi\right|_{\rho=\epsilon}+\delta B \\
& =\left[\delta \varphi_{0} \varphi_{1}+\varphi_{0} \delta \varphi_{1}+2\left(\varphi_{1}+\psi\right) \delta \varphi_{0}+\delta B_{0}\right]+\left[\frac{1}{\epsilon} \varphi_{0} \delta \varphi_{0}+\left(\varphi_{0} \delta \psi+3 \psi \delta \varphi_{0}\right) \log \epsilon+\delta B(\epsilon)\right]
\end{aligned}
$$

The first square brackets corresponds to the variation problem and contain only finite "zero mode" terms. The second bracket contains the divergencies, and represents the "finiteness" problem. We have separated $B$ in two pieces $B(\epsilon)+B_{0}$ stressing its double role. 
We already know the divergencies can be subtracted by $B(\epsilon)$. But let us check this explicitly in this example. The second bracket contains $\psi \delta \varphi_{0}$ which is not an exact variation. However, using

the equation $\psi=-\kappa \varphi_{0}$ it can be written as $\delta\left[\frac{\varphi_{0}^{2}}{2 \epsilon}-2 \kappa \varphi_{0}^{2} \log \epsilon+B(\epsilon)\right]$. This is then removed by choosing $B(\epsilon)=-\frac{\varphi_{0}^{2}}{2 \epsilon}+2 \kappa \varphi_{0}^{2} \log \epsilon$. A less trivial example, gravity in four dimensions, is discussed in Sec. 3.4.

Having discarded the divergencies, we end up with the finite problem, which is the relevant one for the 1-point function. The finite pieces in (19) are almost in the form (9), except for the term $\varphi_{0} \delta \varphi_{1}$. Since the asymptotic equations do not fix $\varphi_{1}$ in terms of $\varphi_{0}$ we cannot express the variation $\delta \varphi_{1}$ in terms of $\delta \varphi_{0}$. At this point $B_{0}$ enters into the game. We choose $B_{0}=-\varphi_{1} \varphi_{0}$ and find,

$$
\delta I=2\left(\varphi_{1}+\psi\right) \delta \varphi_{0} \quad \Rightarrow \quad\langle\mathcal{O}\rangle=2\left(\varphi_{1}+\psi\right)
$$

Two comments are in order. First, the choices made for $B(\epsilon)$ and $B_{0}$ are unique, up to terms local in the source $\varphi_{0}$, which of course cannot be fixed and lead to contact terms. Second, the result (20) is in complete agreement with the method described in [10]. As a final point we note that

$$
B(\epsilon)+B_{0}=-\frac{1}{2 \epsilon^{2}} \Phi^{2}+\frac{2 \kappa \log \epsilon}{\epsilon} \Phi^{2}+\mathcal{O}(\epsilon)
$$

The right hand side of this equation is exactly the counterterm action $C[\Phi]$ that one would have computed following [10].

\subsection{Single massive scalar field}

Scalars fields on the AdS background have been the prototype of system to study and test the AdS/CFT correspondence. We would like to show in this section that variational methods provides strong simplifications in the calculations.

We consider the well known case of a single scalar and reproduce some well-known results. The action we consider is

$$
I=\int d^{d+1} x \sqrt{\hat{g}}\left(\frac{1}{2} \hat{g}^{\mu \nu} \partial_{\mu} \Phi \partial_{\nu} \Phi+\frac{1}{2} m^{2} \Phi^{2}+\right)
$$

This example has been extensively analyzed in the literature. Early calculations [3, 8] did not provide the correct normalization for the vev. This problem was fixed in [4, 9, 10]. 
The field mass becomes related to the conformal dimension $\Delta$ of the dual operator by [3] $m^{2}=$ $\Delta(d-\Delta)$. We follow [10] and consider in this paragraph operators with dimensions

$$
\Delta=\frac{d}{2}+N, \quad N=0,1,2, \ldots
$$

For these masses the asymptotic solution has the Frobenius form with,

$$
\begin{aligned}
\Phi(\rho, x) & =\rho^{\frac{d-\Delta}{2}} \varphi(\rho, x) \\
\varphi(\rho, x) & =\varphi_{0}+\rho \varphi_{1}+\cdots+\rho^{N-1} \varphi_{N-1}+\rho^{N}\left(\varphi_{N}+\psi \log \rho\right)+\cdots
\end{aligned}
$$

The coefficients $\varphi_{1}, \ldots, \varphi_{N-1}$ and $\psi$ are determined in terms of $\varphi_{0}$ by using the asymptotic equations [10]. $\varphi_{N}$ is on the hand undetermined, and is fixed by imposing boundary conditions at $\rho \rightarrow \infty$.

Our goal here is to provide a simple proof of the well-known result,

$$
\langle\mathcal{O}\rangle=(2 \Delta-d) \varphi_{N}+\text { local terms }
$$

using the variational method.

To compute the vev of the dual operator, we vary on-shell the action and plug the series. As shown in Sec. 2.1 we do not need to worry about divergent terms since they can always be renormalized away. The variation of the action is

$$
\begin{aligned}
\delta I & =\int d^{d} x \frac{2}{\rho^{\frac{d}{2}-1}} \Phi^{\prime} \delta \Phi \\
& =2 \int d^{d} x \frac{1}{\rho^{N-1}} \varphi^{\prime} \delta \varphi
\end{aligned}
$$

where we have used (23/24), and discarded a term $\delta\left(\varphi^{2}\right)$. Note that this equation shows clearly why it is necessary to solve the asymptotic equations to order $N$. Now we insert the series (25) and find

$$
\begin{aligned}
\delta I & =2 \int d^{d} x \frac{1}{\rho^{N-1}}\left[\sum_{n}\left(n \rho^{n-1} \varphi_{n}\right)+\rho^{k-1} \psi\right] \sum_{m} \rho^{m} \delta \varphi_{m} \\
& =2 \sum_{n, m} \int d^{d} x \rho^{-N+m+n} n \varphi_{n} \delta \varphi_{m}+\sum_{m} \int d^{d} x \rho^{m} \psi \delta \varphi_{m}
\end{aligned}
$$

In the first line we have already discarded log terms (which remain after computing the derivative in $\varphi^{\prime}$ ) since they cannot contribute to the finite vev; they can be either zero, or divergent in which case they are canceled by a counterterm $B(\rho)$. 
At this point comes the main simplification of our method. The sum in (28) contains $\rho$-dependent terms, and a zero mode term. As explained in Sec. 2.1 we only need to keep the zero mode. Thus we set $m=k-n$ and find

$$
\begin{aligned}
\delta I & =2 \sum_{n} \int d^{d} x n \varphi_{n} \delta \varphi_{k-n}+\psi \delta \varphi_{0} \\
& =2 \int d^{d} x\left[\varphi_{1} \delta \varphi_{N-1}+2 \varphi_{2} \delta \varphi_{N-2}+\cdots+(N-1) \varphi_{N-1} \delta \varphi_{1}+\left(N \varphi_{N}+\psi\right) \delta \varphi_{0}\right]
\end{aligned}
$$

The first $N-1$ terms are purely local because $\varphi_{1}, \ldots, \varphi_{N-1}$ are fixed by the asymptotic equations as functions of $\varphi_{0}$. They can thus be written in the form $X \delta \varphi_{0}$ where $X$ is a local function of $\varphi_{0}$. The last term, on the other hand, contains $\varphi_{N}$ which is non-trivial. Reinserting $N$ in terms of $d$ and $\Delta$ gives the vev (26), as promised.

\subsection{Coulomb branch flow}

In this paragraph we consider another known example [10], namely, gravity coupled to a scalar through the action

$$
I=\frac{1}{4} \int d^{5} x \sqrt{\hat{g}}(\hat{R}-2 \Lambda)-\frac{1}{2} \int d^{4} x \sqrt{h} K+\frac{1}{2} \int d^{5} x \sqrt{\hat{g}}\left(\hat{g}^{\mu \nu} \partial_{\mu} \Phi \partial_{\nu} \Phi+2 V(\Phi)\right)+B+B_{0}
$$

where $V$ is defined by:

$$
V(\Phi)=-2 \Phi^{2}+\frac{4}{3 \sqrt{6}} \Phi^{3}
$$

Since $m^{2}=-4$, the operator dual to $\Phi$ is a scalar operator $O$ whose conformal dimension is $\Delta=2$.

The boundary terms $B(\rho)$ and $B_{0}$ must be chosen such that

$$
\delta I=\int d^{4} x \sqrt{g_{0}}\left[\frac{1}{2}\left\langle T^{i j}\right\rangle \delta g_{0 i j}+\langle O\rangle \delta \phi_{0}\right]
$$

The bulk metric and the scalar take the form [10],

$$
\begin{gathered}
d s^{2}=\frac{d \rho^{2}}{4 \rho^{2}}+\frac{1}{\rho} g_{i j} d x^{i} d x^{j} \quad g_{i j}=g_{0 i j}+\rho g_{1 i j}+\rho^{2}\left(g_{2 i j}+\log \rho h_{1 i j}+(\log \rho)^{2} h_{2 i j}\right)+\ldots \\
\Phi=\rho\left(\tilde{\phi}_{0}+\log \rho \phi_{0}\right)+\rho^{2}\left(\tilde{\phi}_{1}+\log \rho \phi_{1}+(\log \rho)^{2} \psi\right)+\ldots
\end{gathered}
$$

The equations of motion yield,

$$
g_{1 i j}=-\frac{1}{2} R_{0 i j}+\frac{1}{12} R_{0} g_{0 i j} \quad h_{1 i j}=h_{i j}+\frac{2}{3} \phi_{0} \tilde{\phi}_{0} g_{0 i j}
$$




$$
\begin{gathered}
h_{2 i j}=\frac{1}{3} \phi_{0}^{2} g_{0 i j} \quad \operatorname{Trg}_{2}=\frac{1}{4} \operatorname{Tr}\left(g_{1}^{2}\right)+\frac{2}{3}\left(\phi_{0}^{2}+2 \tilde{\phi}_{0}^{2}\right) \\
\phi_{1}=-\frac{1}{4}\left(\square_{0} \phi_{0}+\frac{1}{3} R_{0} \phi_{0}\right)-\frac{4}{\sqrt{6}}\left(\phi_{0}^{2}-\frac{1}{2} \phi_{0} \tilde{\phi}_{0}\right) \\
\tilde{\phi}_{1}=-\frac{1}{4}\left[\square_{0} \phi_{0}+\frac{1}{3} R_{0}\left(\phi_{0}+\tilde{\phi}_{0}\right)+8\left(\phi_{1}+\psi\right)\right]+\frac{1}{\sqrt{6}} \tilde{\phi}_{0}^{2} \\
\psi=\frac{1}{\sqrt{6}} \phi_{0}^{2}
\end{gathered}
$$

where $h_{i j}$ is the value of the log coefficient for the pure gravitational case. Dropping all total variations and divergencies the on-shell variation of the action reads:

$$
\delta I=-\int d^{4} x \sqrt{g} \frac{1}{\rho}\left(k^{i j}-k g^{i j}\right) \delta g_{i j}+2 \int d^{4} x \sqrt{g} \frac{1}{\rho} \Phi^{\prime} \delta \Phi+\delta B+\delta B_{0}
$$

where $k_{i j}=g_{i j}^{\prime}$. Plugging the series (35) and (37) in (38) and choosing properly the boundary terms $B$ and $B_{0}$, is straightforward to find:

$$
\left\langle T^{i j}\right\rangle=t^{i j}+\left[\frac{2}{3} \phi_{0}^{2}+\frac{1}{3} \tilde{\phi}_{0}^{2}-\phi_{0} \tilde{\phi}_{0}\right] g_{0}^{i j}, \quad\langle O\rangle=-2 \tilde{\phi}_{0}
$$

where $t^{i j}$ is the purely gravitational tensor [10]. These results, of course, agree with those found in [10].

\subsection{Gravity and integrability conditions: Einstein equations}

In this section we will consider the case of Gravitation with negative cosmological constant in $d=4$.

The explicit formula for the energy momentum tensor is known [10], and it has also been considered from the variational point of view in [30]. Our goal in this paragraph is to use this system to test the integrability condition (14). The bulk metric takes the form [10],

$$
d s^{2}=\frac{d \rho^{2}}{4 \rho^{2}}+\frac{1}{\rho} g_{i j} d x^{i} d x^{j} \quad g_{i j}=g_{(0) i j}+\rho g_{(1) i j}+\rho^{2}\left(g_{(2) i j}+\log \rho h_{i j}\right)+\ldots
$$

The values of $g_{(1) i j}, h_{i j}, \operatorname{Tr} g_{(2) i j}$ and Div $g_{(2)}$ are local functions of $g_{(0) i j}$. In particular,

$$
g_{(1) i j}=\frac{1}{12} R_{(0)} g_{(0) i j}-\frac{1}{2} R_{(0) i j} .
$$

This value is universal and valid for any gravitational action that accepts an AdS background [24]. 
In this paragraph we only want to address the divergencies in the variation of the action (for the finite part see [30]). After subtracting total variations, there remains two divergent terms,

$$
\delta I_{d i v}=-\int \sqrt{g(0)}\left[\frac{1}{\rho}\left(g_{(1)}^{i j}-\operatorname{Tr}\left(g_{1}\right) g_{(0)}^{i j}\right)+\frac{1}{2} \log \rho h^{i j}\right] \delta g_{(0) i j} .
$$

According to the general discussion in Sec. 2.1, these terms can be written as total variations. In fact, it is known [10] that $h^{i j}$ can be written as a total "divergency",

$$
h^{i j}=\frac{\delta}{\delta g_{(0) i j}} \int \mathcal{A}
$$

where $\mathcal{A}$ is the anomaly. This shows that the log divergency can in fact be written as a total variation. On the other hand, from (41) it is direct to see that $\left(g_{(1)}^{i j}-\operatorname{Tr}\left(g_{(1)}\right) g_{(0)}^{i j}\right)$ is proportional to the Einstein tensor, and thus, the $1 / \rho$ divergency can also be written as a total variation $\delta(\sqrt{g} R)$. These counterterms were first derived in [34] by demanding finiteness of the energy-momentum tensor.

\section{Higher order theories: $L=L\left(q, q^{\prime}, q^{\prime \prime}, \ldots\right)$}

We have shown in this paper that for any Lagrangian $L\left(\phi, \phi^{\prime}\right)$ the divergencies appearing in the variation of the action can be canceled by a boundary term. We would like to show in this paragraph that the same conclusions follow with higher order theories with Lagrangians of the form $L\left(\phi, \phi^{\prime} \phi^{\prime \prime}, \ldots\right)$. This means that our results are applicable to string theories with higher $\alpha^{\prime}$ corrections.

Let us work out here the details for a fourth order theory with $L\left(\phi, \phi^{\prime}, \phi^{\prime \prime}\right)$. The variation of the action yields,

$$
\delta I=\int\left[\left(\frac{\partial L}{\partial \phi^{\prime \prime}}\right)^{\prime \prime}-\left(\frac{\partial L}{\partial \phi^{\prime}}\right)^{\prime}+\frac{\partial L}{\partial \phi}\right] \delta \phi+[\underbrace{\frac{\partial L}{\partial \phi^{\prime}} \delta \phi+\frac{\partial L}{\partial \phi^{\prime \prime}} \delta \phi^{\prime}-\left(\frac{L}{\phi^{\prime \prime}}\right)^{\prime} \delta \phi}]_{\rho=\epsilon}
$$

The first parenthesis gives the Euler-Lagrange equations while the second is a boundary term. We shall prove now that the $\rho$-dependent parts of this boundary term can be written as a total variation, and hence subtracted from the action. Let us denote the piece enclosed by the underbrace as $B$. We proceed exactly as in the second order case. Computing the radial derivative of $B$ and using the equations of motion we find,

$$
B^{\prime}=\left(\frac{\partial L}{\partial \phi^{\prime}}\right)^{\prime} \delta \phi+\frac{\partial L}{\partial \phi^{\prime}} \delta \phi^{\prime}+\frac{\partial L}{\partial \phi^{\prime \prime}} \delta \phi^{\prime \prime}-\left(\frac{\partial L}{\partial \phi^{\prime \prime}}\right)^{\prime \prime} \delta \phi
$$




$$
\begin{aligned}
& =\frac{\partial L}{\partial \phi} \delta \phi+\frac{\partial L}{\partial \phi^{\prime}} \delta \phi^{\prime}+\frac{\partial L}{\partial \phi^{\prime \prime}} \delta \phi^{\prime \prime} \\
& =\delta L .
\end{aligned}
$$

As in the second order theory, this shows that all divergencies present in $B$ can be renormalized away. The zero mode of $B$ is not restricted by the equation. For higher order theories the boundary data has a bigger structure (more initial conditions) and hence the AdS/CFT dictionary may need extra revision.

\section{Acknowledgments}

We would like to thank S. Theisen, M. Henneaux and C. Nuñez for their useful comments and discussions. We also thank M. Berg, M. Haack and W. Mück for useful comments on an earlier version of this manuscript. M.B. work is partially supported by FONDECYT \# 1060648.

\section{References}

[1] J. Maldacena, Adv. Theor. Math. Phys. 2, 231 (1998) arXiv:hep-th/9711200.

[2] S. Gubser, I. Klebanov, A. Polyakov, Phys. Lett. B 428, 105 (1998) arXiv:hep-th/9802109].

[3] E. Witten, Adv.Theor.Math.Phys 2, 253 (1998) arXiv:hep-th/9802150].

[4] I. R. Klebanov, E. Witten, Nucl.Phys.B 556, 89 (1999) arXiv:hep-th/9905104.

[5] E. Witten, arXiv:hep-th/0112258.

[6] M. Henneaux, arXiv:hep-th/9902137.

[7] M. Henningson, K. Sfetsos, Phys.Lett.B 431, 63 (1998) arXiv:hep-th/9803251.

[8] D. Z. Freedman, S. D. Mathur, A. Matusis and L. Rastelli, Nucl. Phys. B 546, 96 (1999) arXiv:hep-th/9804058.

[9] W. Mück and K. S. Viswanathan, Phys. Rev. D 58, 106006 (1998) arXiv:hep-th/9805145. W. Mück and K. S. Viswanathan, Phys. Rev. D 58, 041901 (1998) arXiv:hep-th/9804035]. 
[10] S. de Haro, S. Solodukhin, K. Skenderis, Commun.Math.Phys 217, 595 (2001) |arXiv:hep-th/0002230]. M. Bianchi, D. Z. Freedman and K. Skenderis, JHEP 0108, 041 (2001) |arXiv:hep-th/0105276]. M. Bianchi, D. Z. Freedman, K. Skenderis, Nucl.Phys.B 631, 159 (2002) arXiv:hep-th/0112119.

[11] G. E. Arutyunov and S. A. Frolov, Nucl. Phys. B 544, 576 (1999) arXiv:hep-th/9806216.

[12] G. Arutyunov, S. Frolov and S. Theisen, Phys. Lett. B 484, 295 (2000) arXiv:hep-th/0003116].

[13] P. Minces and V. O. Rivelles, JHEP 0112, 010 (2001) arXiv:hep-th/0110189. P. Minces and V. O. Rivelles, Nucl. Phys. B 572, 651 (2000) arXiv:hep-th/9907079.

[14] H. Boschi-Filho and N. R. F. Braga, Phys. Lett. B 471, 162 (1999) arXiv:hep-th/9910233.

[15] D. Nolland, Phys. Lett. B 584, 192 (2004) arXiv:hep-th/0310169].

[16] W. Mück, Phys. Lett. B 531, 301 (2002) arXiv:hep-th/0201100].

[17] T. Hartman and L. Rastelli, arXiv:hep-th/0602106.

[18] M. Berkooz, A. Sever and A. Shomer, JHEP 0205, 034 (2002) arXiv:hep-th/0112264.

[19] I. Papadimitriou, arXiv:hep-th/0606038.

[20] D. Marolf and S. Ross, JHEP 0611, 085 (2006) arXiv:hep-th/0606113.

[21] P. Minces, Phys. Rev. D 68, 024027 (2003) arXiv:hep-th/0201172.

[22] I. Papadimitriou, K. Skenderis, arXiv:hep-th/0404176]. I. Papadimitriou, K. Skenderis, JHEP 0410, 075 (2004) arXiv:hep-th/0407071

[23] I. Papadimitriou and K. Skenderis, JHEP 0508, 004 (2005) arXiv:hep-th/0505190.

[24] C. Imbimbo, A. Schwimmer, S. Theisen, S. Yankielowicz, Class.Quant.Grav. 17, 1129, (2000) arXiv:hep-th/9910267.

[25] A. Schwimmer, S. Theisen, JHEP 0008, 32 (2000) arXiv:hep-th/0008082.

[26] A. Schwimmer, S. Theisen, JHEP 0310, 001 (2003) arXiv:hep-th/0309064. 
[27] J. de Boer, E. P. Verlinde and H. L. Verlinde, JHEP 0008, 003 (2000) arXiv:hep-th/9912012].

[28] M. Henningson, K. Skenderis, JHEP 9807, 023 (1998) arXiv:hep-th/9806087.

[29] M. Berg, M. Haack and W. Mück, Nucl. Phys. B 736, 82 (2006) arXiv:hep-th/0507285].

[30] M. Bañados, A. Schwimmer, S. Theisen, JHEP 0405, 039, (2004) arXiv:hep-th/0404245].

[31] M. Bañados, R. Olea, S. Theisen, JHEP 0510, 067 (2005) [arXiv:hep-th/0509179]. M. Bañados, O. Miskovic, S. Theisen, JHEP 0606, 025, (2006) arXiv:hep-th/0604148].

[32] J. Gegenberg, C. Martínez, R. Troncoso Phys. Rev. D 67, 084007 (2003) arXiv:hep-th/0301190 M. Henneaux, C. Martínez, R. Troncoso and J. Zanelli, Phys. Rev. D 70, 044034 (2004) arXiv:hep-th/0404236. T. Hertog and K. Maeda, JHEP 0407, 051 (2004) arXiv:hep-th/0404261]. M. Henneaux, C. Martínez, R. Troncoso and J. Zanelli, arXiv:hep-th/0603185.

[33] M. Banados, A. Schwimmer and S. Theisen, JHEP 0609, 058 (2006) arXiv:hep-th/0604165.

[34] V. Balasubramanian and P. Kraus, Commun. Math. Phys. 208, 413 (1999) arXiv:hep-th/9902121. 